\title{
Induction of the chemokine stromal-derived factor-1 following DNA damage improves human stem cell function
}

\author{
Tanya Ponomaryov, ${ }^{1}$ Amnon Peled, ${ }^{1}$ Isabelle Petit,${ }^{1}$ Russell S. Taichman, ${ }^{2}$ \\ Liliana Habler, ${ }^{3}$ Judith Sandbank, ${ }^{3}$ Fernando Arenzana-Seisdedos, ${ }^{4}$ \\ Aude Magerus, ${ }^{4}$ Antonio Caruz, ${ }^{5}$ Nobutaka Fujii, ${ }^{6}$ Arnon Nagler, ${ }^{7}$ \\ Meir Lahav, ${ }^{8}$ Martin Szyper-Kravitz, ${ }^{8}$ Dov Zipori, ${ }^{9}$ and Tsvee Lapidot ${ }^{1}$
}

${ }^{1}$ Department of Immunology, The Weizmann Institute of Science, Rehovot, Israel

${ }^{2}$ University of Michigan Dental School, Department of Periodontics, Ann Arbor, Michigan, USA

${ }^{3}$ Assaf Harofeh Tsrifin Medical Center, Tsrifin, Israel

${ }^{4}$ Institute Pasteur, Paris, France

${ }^{5}$ University of Jaen, Jaen, Spain

${ }^{6}$ University of Kyoto, Kyoto, Japan

${ }^{7}$ Hadassah University Hospital, Jerusalem, Israel

${ }^{8}$ Meir Medical Center, Kfar-Saba, Israel

${ }^{9}$ Department of Molecular Cell Biology, The Weizmann Institute of Science, Rehovot, Israel

Address correspondence to: Tsvee Lapidot, Department of Immunology, The Weizmann Institute of Science,

PO Box 26, Rehovot 76100, Israel. Phone: 972-8-9342481; Fax: 972-8-9344141; E-mail: Tsvee.Lapidot@weizmann.ac.il.

Received for publication May 15, 2000, and accepted in revised form October 11, 2000.

The chemokine stromal-derived factor-1 (SDF-1) controls many aspects of stem cell function. Details of its regulation and sites of production are currently unknown. We report that in the bone marrow, SDF-1 is produced mainly by immature osteoblasts and endothelial cells. Conditioning with DNAdamaging agents (ionizing irradiation, cyclophosphamide, and 5-fluorouracil) caused an increase in SDF-1 expression and in CXCR4-dependent homing and repopulation by human stem cells transplanted into NOD/SCID mice. Our findings suggest that immature osteoblasts and endothelial cells control stem cell homing, retention, and repopulation by secreting SDF-1, which also participates in host defense responses to DNA damage.

J. Clin. Invest. 106:1331-1339 (2000).

\section{Introduction}

Hematopoietic stem cells reside within the bone marrow (BM) microenvironment in direct contact with stromal cells. The latter regulate the continuous production of maturing blood cells and their release into the blood circulation while maintaining a small pool of primitive, undifferentiated stem cells (1). During development, blood-forming stem cells migrate from the fetal liver into the circulation and home to the BM microenvironment (2). Similarly, in clinical and experimental stem cell transplantation, intravenously injected stem cells migrate through the blood and home to the BM, repopulating it with immature and maturing myeloid and lymphoid blood cells (3). Functional in vivo assays for human stem cells based on transplantation into immune-deficient SCID, and more recently into NOD/SCID or B2m-null NOD/SCID mice, have been developed by us, as well as in other laboratories (4-7). These assays identified a small population of primitive human severe combined immunodeficiency (SCID) repopulating cells (SRC), by virtue of their functional ability to repopulate transplanted recipients with both myeloid and lymphoid cells (8). Determining the role of stromal cells in stem cell homing, retention, and repopulation will help to identify the mechanisms that govern stem cell development and may advance clinical applications to stem cell transplantation.
Stromal-derived factor-1 (SDF-1), also known as pre-B-cell growth-stimulating factor, is produced in many organs, including the BM $(2,9)$. However, details of its expression pattern and mode of regulation are currently unknown. In contrast to proinflammatory chemokines, SDF-1 is produced by BM stromal cells also in the absence of stimuli generated by viral or bacterial infections, suggesting a major role for SDF-1 in steady-state homeostatic processes such as leukocyte trafficking $(2,9)$. In accordance with this notion has been the identification of SDF-1 as a powerful chemoattractant for immature and mature hematopoietic cells of several lineages $(2,10-12)$. Mice that lack either SDF-1 or its receptor CXCR4 exhibit many defects, including impaired hematopoiesis in the fetal $\operatorname{BM}(9,13)$. Recently, we discovered that human stem cell engraftment of NOD/SCID mice is dependent on the expression of SDF-1 and CXCR4 (14). Interestingly, the BM of mice transplanted with fetal liver cells from CXCR4-deficient mice was repopulated by donor cells $(15,16)$. However, release of differentiating cells from the marrow into the circulation and the levels of donor-derived hematopoiesis in the BM of these mice was significantly reduced. These results demonstrate that long-term repopulation by pluripotent stem cells is impaired in CXCR4-null fetal liver cells, since these 
cells fail to durably proliferate and differentiate in the absence of CXCR4. In support of this notion, recent studies demonstrated that SDF-1 is a survival factor for both murine and human immature progenitor cells (17-19). Other studies indicate that SDF-1 is a pre-Bcell growth factor, that it is also involved in the development of megakaryocytes, that it is an inhibitor of myelopoiesis, and that it prevents primitive human cells from entering the cell cycle (20-23). We have demonstrated that SDF-1 plays a pivotal role in the conversion of $\mathrm{CD} 34^{+}$cell-rolling adhesions on endothelial cells into firm adhesions by activating VLA- 4 and LFA-1 (24). Furthermore, it has been shown that SDF-1 is expressed by human and murine BM endothelium in vivo, while murine endothelial cells from the lung or human umbilical cord did not express the chemokine $(24,25)$. Activation of LFA-1, VLA-4, and VLA- 5 on human CD $34^{+}$cells by SDF- 1 is crucial for their engraftment in transplanted NOD/SCID (26). Lastly, preliminary results indicate that CXCR4-null fetal liver cells can also interact with SDF-1 independently of CXCR4, which partially compensates for the absence of signaling via CXCR4 (27). In support of our work, adult mice reconstituted with genetically modified BM cells with the SDF-1 intrakine gene, had significantly reduced levels of both lymphoid pre-B cells and myeloid cells in their BM (28).

SDF-1 is thus important in stem cell trafficking and development. It is not known, however, which $\mathrm{BM}$ cells are primarily responsible for the production of SDF-1, nor is it known how SDF-1 expression is regulated. The hematopoietic system is the most sensitive to DNA-damaging agents, such as irradiation or cytotoxic drugs. This vulnerability of bloodforming stem cells reduces regeneration after treatment or accidental exposure to these agents and thus impinges on long-term survival (29). In the present study, we identified BM cell types that express SDF-1 in vivo and in vitro. We further tested the effect of DNA-damaging agents such as irradiation, cyclophosphamide (Cy), or 5-fluorouracil (5-FU) on the expression of SDF-1 in the BM of mice or in cultured stromal cells. In parallel, we determined the time-dependent effect of irradiation or other DNA-damaging agents on the CXCR4-dependent repopulation potential of human stem cells in transplanted NOD/SCID mice.

\section{Methods}

Human and mouse cells. Human cord blood (CB) cells were obtained from full-term deliveries after informed consent and were used in accordance with approved procedures by the human experimentation and ethics committees of the Weizmann Institute. CB samples were diluted in PBS, supplemented with 1\% FCS (Biological Industries, Beit Haemek, Israel). Low-density mononuclear cells were collected after standard separation on Ficoll-Paque (Pharmacia Biotech AB, Uppsala, Sweden), and washed with PBS.
Enrichment of buman $\mathrm{CD} 34^{+}$cells. Enrichment of human $\mathrm{CD}^{+} 4^{+}$cells was performed with a magnetic bead separation kit (mini MACS; Miltenyi Biotec, Bergisch Gladbach, Germany) according to the manufacturer's instructions. The purity of the enriched $\mathrm{CD} 34^{+}$cells was 60-85\% when cells were passed over one column, and greater than $98 \%$ when passed over two columns.

Human umbilical vein endothelial cells (HUVECs) were isolated from umbilical cord veins, according to the method of Jaffe et al. (30). Human adherent stromal cells were prepared and grown as described previously from leftover BM cells for allogeneic transplantation (31). Mouse BM stromal-cell lines (MBA-2.1, 14F1.1, MBA-15) were grown as described previously $(32,33)$. U373-MG, a human glioblastoma cell line (ATTC number HTB17; American Type Culture Collection, Rockville, Maryland, USA), was cultured in DMEM. MS-5, a murine stromal cell line, is a kind gift from J.C. Gutierrez-Ramos (Millennium Pharmaceuticals, Cambridge, Massachusetts, USA). Cells were grown in RPMI1640 complemented with $50 \mathrm{nM} 2$-mercaptoethanol. Both culture media were enriched with $10 \%$ FCS. Conditioned media (CM) from confluent layers of murine and human stroma cells was collected after 3 days in culture. Mouse CM were prepared from supernatants containing single-cell suspensions of flushed murine $\mathrm{BM}$ and spleen cells in RPMI with 10\% FCS after centrifugation and storage at $-20^{\circ} \mathrm{C}$.

Osteosarcoma cell lines and primary buman osteoblast-like cells. Human osteosarcoma cell lines were purchased from the American Tissue Type Collection, including MG-63 (ATCC CRL1424) and SaOS-2 (ATCC 85-HTB). These cell lines were maintained in either DMEM with Earle's salts (HOS and MG-63) or McCoy's 5A medium (G-292, SaOS-2, U2-OS) with 10\% heat-inactivated FBS and antibiotics. Enriched human osteoblast-like cell (HOB) cultures were established using modifications of methods described by Robey and Termine (34). Normal human trabecular bone was obtained from patients undergoing orthopedic surgery in accordance with the University of Michigan's Investigational Review Board. Bone cleaned of loosely adherent tissue was ground to produce a uniform particle size (size $\leq 1$ $\mathrm{mm}^{2}$ ) (BioComp Minimill; Walter Lorenz Surgical Inc., Jacksonville, Florida, USA) and incubated in $1 \mathrm{mg} / \mathrm{ml}$ bacterial collagenase (Type P; Boehringer Mannheim Biochemicals Inc., Indianapolis, Indiana, USA). The explants were placed into culture until confluent monolayers were produced in a $1: 1(\mathrm{vol} / \mathrm{vol})$ mixture of Ham's F12/DMEM (Biofluids, Rockville, Maryland, USA) with low $\mathrm{Ca}^{2+}$ and $10 \%$ heat-inactivated FBS. Thereafter, the cultures were maintained in calciumreplete Ham's F12/DMEM (1:1 vol/vol) medium containing $10 \%$ heat-inactivated FCS, antibiotics $10 \mathrm{mM}$ $\beta$-glycerol phosphate and $10 \mu \mathrm{g} / \mathrm{ml} \mathrm{L}$-ascorbate. To verify that the cells expressed an osteoblast phenotype, the cultures were screened for the expression of the osteoblast-specific protein osteocalcin by RT-PCR, as detailed previously (35). 
Mice. NOD-SCID mice (NOD/LtSzPrKdc scid $/$ PrKdc scid) were bred and maintained under defined flora conditions in individually ventilated (HEPA-filtered air) sterile micro-isolator cages (Techniplast, Gazzada, Italy) at the Weizmann Institute. All the experiments were approved by the Animal Care Committee of the Weizmann Institute. Mice, 8 weeks old, were irradiated with a sublethal dose of $375 \mathrm{cGy}$ (67 cGy/min.) from a cobalt source before transplantation or treatment with Cy (200 $\mathrm{mg} / \mathrm{kg})$ or 5 -FU $(200-250 \mathrm{mg} / \mathrm{kg})$. Human CD34+enriched cells (60-85\% purity, $2 \times 10^{5} /$ mouse) or mononuclear cells $\left(1-2 \times 10^{7} /\right.$ mouse $)$ were injected into the tail veins of mice irradiated immediately, or 48 hours earlier, in $0.5 \mathrm{ml}$ of RPMI with $10 \%$ FCS. Transplanted mice were sacrificed after 4-5 weeks or the following day in homing experiments, and the cells from the spleen, femur, tibia, and pelvis bones were flushed with a syringe. BALB/c and C57BL mice were purchased from Harlan Laboratories (Rehovot, Israel). Mice were injected intraperitoneally with Cy $(200 \mathrm{mg} / \mathrm{kg})$ or 5 -FU $(150 \mathrm{mg} / \mathrm{kg})$ and sacrificed at the indicated time points.

Semiquantitative RT-PCR. Total RNA was isolated from MBA-2.1, 14F1.1, and MBA-15 cells (with or without treatment with $150 \mu \mathrm{g} / \mathrm{ml} \mathrm{5-FU} \mathrm{or} 200 \mu \mathrm{g} / \mathrm{ml} \mathrm{Cy}$ ), human stromal cells, and mice tissues (BM and spleen) using TRI-Reagent (Molecular Research Center Inc., Cincinnati, Ohio, USA) according to the manufacturer's protocol. Each RNA sample $(1 \mu \mathrm{g})$ was subjected to cDNA synthesis in $30 \mu \mathrm{l}$ of reaction mixture containing $1 \mu \mathrm{l}$ Oligo dT 15 primer $(500 \mu \mathrm{g} / \mathrm{ml}$; Promega Corp., Madison, Wisconsin, USA), $2 \mu \mathrm{l}$ dNTP's mixture (10 mM, PCR grade; Boehringer Mannheim Biochemicals Inc.), $3 \mu \mathrm{l}$ DTT (0.1 M; Promega Corp.), $1 \mu \mathrm{l}$ RNasin (40 $\mathrm{U} / \mu \mathrm{l}$; Promega Corp.) and $1 \mu \mathrm{l}$ MMLV-RT $(200 \mathrm{U} / \mu \mathrm{l}$; Promega Corp.) in the supplied reaction buffer $(5 \times, 250$ mM Tris- $\mathrm{HCl}, \mathrm{pH} 8.3,375$ mM KCl, 15 mM MgCl2, 50 mM DTT; Promega Corp.) for 1 hour at $42^{\circ} \mathrm{C}$. The PCR was performed in a $50-\mu \mathrm{l}$ reaction mixture using $5 \mu \mathrm{l}$ of cDNA, Taq DNA Polymerase (Promega Corp.), $1 \mu \mathrm{l}$ of dNTP's mixture (10 mM; Boehringer Mannheim Biochemicals Inc.), and specific primers for 35 cycles at appropriate annealing temperatures. As a control for primer contamination or dimerization, the same reaction mixture without cDNA was prepared. Semiquantitative analysis for SDF-1 expression in murine tissues and cell lines was performed for 25 cycles: Tann $=65^{\circ} \mathrm{C}$, 45 seconds. The resulting PCR products were separated on $1.6 \%$ agarose gel (SeaKem LE agarose; FMC Corp., Rockland, Maine, USA).

Semiquantitative RT-PCR for HOBs and osteosarcoma cell lines. Cells were plated at an initial density of $2 \times 10^{5}$ cells $/ \mathrm{cm}^{2}$ with complete medium changes in medium containing freshly prepared $\beta$-glycerol phosphate (10 $\mathrm{mM})$ and L-ascorbate $(50 \mu \mathrm{g} / \mathrm{ml})$ on days $5,7,10$, and 14 . One hundred fifty micrograms per milliliter 5-FU (Sigma Chemical Co., St. Louis, Missouri, USA) was added to the cultures on day 10 . On day 14 , total cellular RNA was recovered, reverse transcribed, and PCR was performed at $94^{\circ} \mathrm{C}$ for 1 minute, $60^{\circ} \mathrm{C}$ for 1 minute, and $72^{\circ} \mathrm{C}$ for 1 minute for 27 or 35 cycles (semiquantitative and nonquantitative, respectively, followed by a 10 minute extension at $72^{\circ} \mathrm{C}$ (Cetus DNA thermal cycler; Perkin Elmer Applied Biosystems, Foster City, California, USA) as described previously with sense and antisense primers prepared by the oligonucleotide synthesis core at the University of Michigan (36).

Primer sequences and lengths of products. Primer sequences used were: murine SDF-1 (5' GGA CGC CAA GGT CGT CGC CGT G, 3’ TTG CAT CTC CCA CGG ATG TCA G, 335 bp); murine CXCR4 (5' GAC GGA CAA GTA CCG GCT GC, 3’ GAC AGC TTA GAG ATG ATG AT, 482 bp); human SDF-1 (5' CGT CAG CCG CAT TGC CCG CT, 3' GGT CTA GCG GAA AGT CCT, 380 bp); human CXCR4 (5' CTG AGA AGC ATG ACG GAC AA, 3' TGG AGT GTG ACA GCT TGG AG, 484 bp); GADPH (5' GAC AAC AGC CTCAAG ATC ATC AGC, 3’ AAG TCA GAG GAG ACC ACC TGG TGC); $\beta$-actin ( $5^{\prime}$ TCC TGT GGC ATC CAT GAA ACT ACA TTC AAT TCC, 3' GTG AAA ACG CAG CTC AGT AAC AGT CCG CCT AG, 347 bp).

Flow-cytometry analysis of human cells. In mouse BM or spleen: for immunostaining, approximately $10^{5}$ cells were resuspended in staining buffer (PBS, $0.1 \%$ BSA, $0.02 \%$ sodium azide), incubated with $10 \mu \mathrm{g} / \mathrm{ml}(1: 50)$ of purified anti-mouse CD16/CD32 (FcR) (PharMingen, San Diego, California, USA) and $1 \%$ human plasma for 20 minutes at $4^{\circ} \mathrm{C}$. Cells were then stained with human-specific, direct-labeled Ab's and incubated for 30 minutes on ice. Dead cells were gated out by staining with propidium iodide (Sigma Chemical Co.). Human cells from engrafted mice were analyzed by double-staining with anti-CD45 FITC (Immuno Quality Products, Gromingen, The Netherlands) and antiCD19 phycoerythrin (PE) (lymphoid) or anti-CD33 (myeloid) (Coulter, Miami, Florida, USA). The levels of immature cells in the Transwell migration assay were analyzed by double-staining with anti-CD34 FITC (Becton Dickinson Immunocytometry Systems, San Jose, California, USA) and anti-CD38PE (Coulter). After staining, cells were washed twice in the same buffer and analyzed on a FACSort (Becton Dickinson Immunocytometry Systems), using CellQuest software (Becton Dickinson Immunocytometry Systems).

Cell sorting. Cell sorting was performed on a FACStar plus (Becton Dickinson Immunocytometry Systems) as described previously $(8,14)$. In brief, single-cell suspensions of human CD34+-enriched cells (MiniMacs) were labeled with anti-human CD34 FITC (Becton Dickinson Immunocytometry Systems) and anti-human CD38 PE (Coulter) mAb's. The purity of sorted CD34 $/ \mathrm{CD} 38^{-/ \text {low }}$ and $\mathrm{CD}_{3} 4^{+} / \mathrm{CD} 38^{+}$cells was greater than $99 \%$.

Chemotaxis assay. Chemotaxis experiments with human $\mathrm{CD} 4^{+}$cells were assayed by using Costar Transwells (6.5 mm/diameter, $5 \mathrm{~mm} /$ pore; Corning Costar, Cambridge, Massachusetts, USA). One hundred microliters of chemotaxis buffer (RPMI-1640, 1\% FCS) containing $2 \times 10^{5}$ cells were added in the upper chamber, and $0.6 \mathrm{ml}$ of either chemotaxis buffer both with or without $125 \mathrm{ng} / \mathrm{ml}$ of SDF-1 or condition media was 

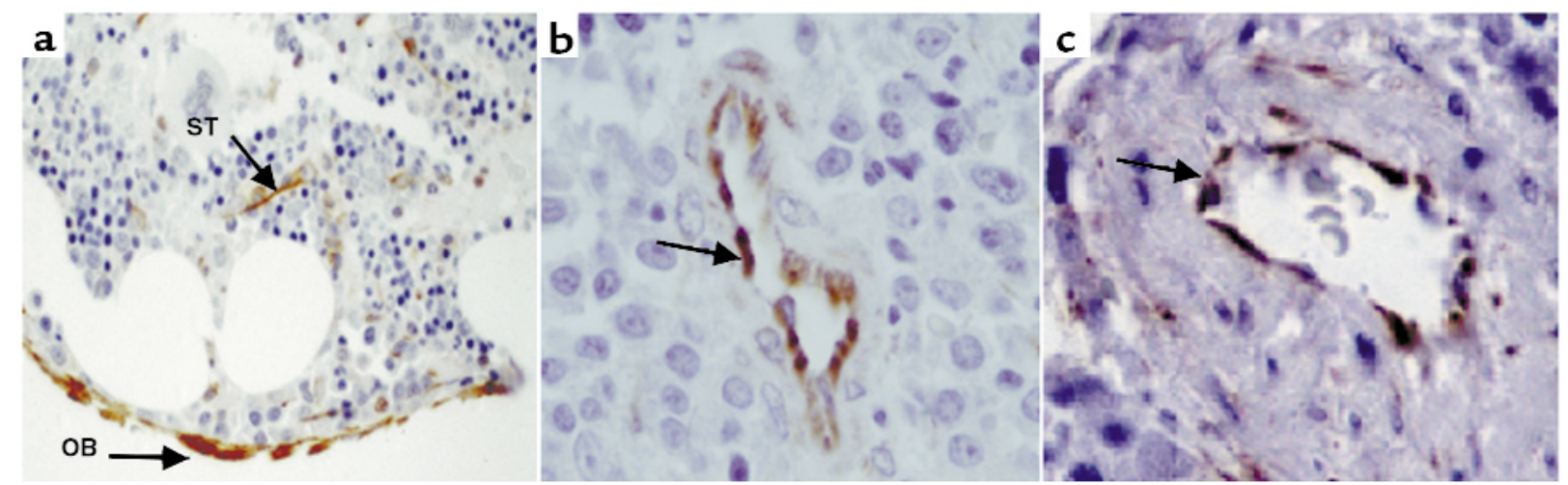

Figure 1

Anti-SDF-1 reactive immature BM osteoblasts lining the endosteum region (OB) and stromal cells (ST; arrows, $\times 200)($ a), venules (arrow) (b), and arteriole (arrow) (c) in human BM sections $(\times 1000)$. No peroxidase staining of control mAb was observed on identical tissue sections (data not shown).

added to the bottom chamber. Cells migrating within 4 hours to the bottom chamber of the Transwell were counted for 30 seconds using a FACSort (Becton Dickinson Immunocytometry Systems). SDF-1 was purchased from R\&D Systems Inc. (Minneapolis, Minnesota, USA). For the inhibition of chemotaxis the preincubation of $\mathrm{CD} 34^{+}$cells with anti-CXCR $4 \mathrm{mAb}$ 12g5 (PharMingen) or T22 CXCR4-binding peptide was performed for 30 minutes before the migration.

Colony assay. Semisolid progenitor cultures were performed as described previously (5). In brief, the cells were plated in $0.9 \%$ methylcellulose (Sigma Chemical Co.), $30 \%$ FCS, $5 \times 10^{-5} 2 \mathrm{ME}$ (Sigma Chemical Co.), $50 \mathrm{ng} / \mathrm{ml}$ stem cell factor (SCF), $5 \mathrm{ng} / \mathrm{ml} \mathrm{IL-3,} 5 \mathrm{ng} / \mathrm{ml} \mathrm{GM-CSF} \mathrm{(R \& D}$ Systems Inc.), and $2 \mathrm{U} / \mathrm{ml}$ erythropoietin (EPO; Orto Bio Tech, Don Mills, Ontario, Canada). BM cells from transplanted mice were cultured under conditions selected for growth of human colonies only by replacing 15\% FCS with $15 \%$ human plasma. Plating concentrations for enriched $\mathrm{CD} 34^{+}$cells were $3 \times 10^{3}$ cells $/ \mathrm{ml}$, and for $\mathrm{BM}$ cells from transplanted mice, $200 \times 10^{3}$ cells $/ \mathrm{ml}$. The cultures were incubated at $37^{\circ} \mathrm{C}$ in a humidified atmosphere containing $5 \% \mathrm{CO}_{2}$ and were scored 14 days later.

Immunohistology of BM sections. Immunohistochemical staining was performed on formalin-fixed, paraffinembedded human bone sections, using the avidin-biotinperoxidase-complex method and diaminobenzidine tetrahydrochloride (DAB) chromogen kit (DAKO A/S, Glostrup, Denmark). Human bone sections were stained with the anti-SDF-1 mAb K15C (1:50 dilution; refs. 24, 37). The immunohistochemical staining was performed using the $\mathrm{LSAB}^{+}$peroxidase kit (DAKO A/S). To enhance immunostaining, the antigen was preretrieved in citrate buffer using pressure cooking within a calibrated microwave, heated for 15 minutes, and then reheated for additional 2.5 minutes. Slides were counterstained with Mayer's hematoxylin. The specificity of the anti-SDF-1 $\mathrm{mAb}$ was confirmed by the complete absence of peroxidase staining on identical tissue sections using an isotype-matched control $\mathrm{mAb}$ (data not shown). a Murine and human
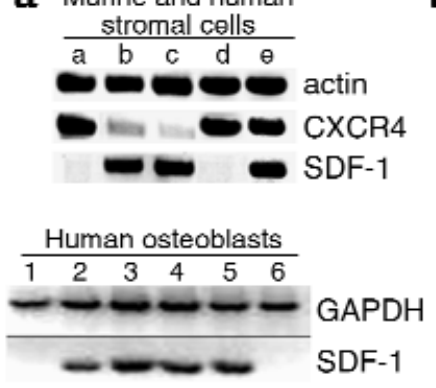

b

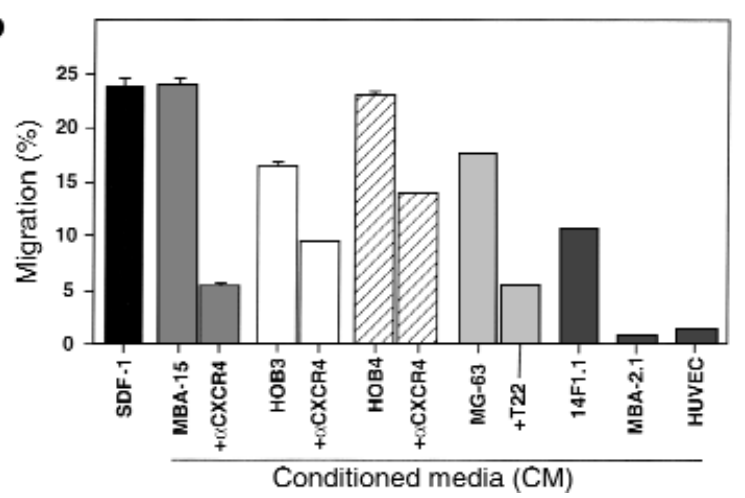

c

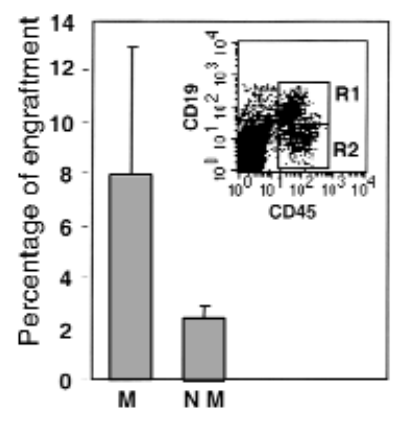

\section{Figure 2}

(a) Expression of SDF-1 and CXCR4 by RT-PCR. Lane a: endothelial MBA-2.1 cells; lane b, adipocyte 14F1.1 cells; lane c, osteoblast MBA15 cells; lane d, HUVECs; lane e, primary human stroma. Expression of GAPDH and SDF-1: primary HOBs (lanes 1-4), osteosarcomas MG63 (lane 5), and SaOS-2 (lane 6). (b) Migration of CD34+ cells to CM: SDF-1 migration to $125 \mathrm{ng} / \mathrm{ml} \mathrm{SDF-1,} \mathrm{MBA-15,} \mathrm{HOB3,} \mathrm{HOB4,} \mathrm{MG-}$ 63, 14F1.1, MBA-2.1, HUVECs. $\alpha$ CXCR4 or T22 pretreatment of CD34+ before Transwell migration. The results are the mean of seven different experiments \pm SE. (c) Engraftment by CD34 cells migrating (M) and nonmigrating (NM) to MBA15 CM. Human CD45 ${ }^{+} \mathrm{CD} 19^{+}$ cells (R1) in the BM of mice transplanted with cells migrating to CM of MBA-15. 
ELISA. The amount of SDF-1 released in the cell-culture supernatants was estimated by ELISA. SDF-1 was captured by an anti-human/mouse monoclonal SDF-1 $\mathrm{Ab}$ (Research Diagnostics Inc., Flanders, New Jersey, USA). Immobilized SDF-1 was detected using a biotinylated polyclonal SDF-1 Ab (R\&D Systems Inc.) coupled to horseradish peroxidase-labeled streptavidin.

\section{Results}

SDF-1 is bighly expressed by buman BM osteoblasts and endothelial cells. The potential role of SDF-1 in human stem and progenitor cell migration and localization in the $\mathrm{BM}$ has been studied by staining human bone tissue sections with mAb specific for $\operatorname{SDF}-1(24,37)$. We found that SDF-1 is produced mainly by immature osteoblasts lining the bone endosteum region (Figure 1a) and by endothelial cells lining the small and large vessels, including periarterial regions (Figure 1, b and c) as well as the blood capillaries of the bone (24). Stromal cells in the mesenchyme were also stained by the $\mathrm{Ab}$ (Figure 1a). We next tested the expression and production of SDF-1 in different cell lines representing various murine BM stroma cell types. By RT-PCR analysis we found that murine cell lines with osteoblastic characteristics such as MBA-15 (Figure 2a, lane c), and most HOBs (Figure 2a, lanes 1-4) and/or human osteosarcomas (Figure 2a, lanes 5 and 6), express high levels of SDF-1 mRNA. SDF-1 is also expressed by murine mesenchymal adipocyte 14F1.1 cells (lane b) and by primary human stromal cells (lane e) (Figure 2a). Interestingly, BM-derived murine endothelial cells (MBA-2.1, lane a), as well as the HUVECs (lane d), did not express SDF-1 (Figure 2a). CXCR4, the receptor for SDF-1, has been shown to be functionally expressed by endothelial cells such as HUVECs (38). We found that CXCR4 was not expressed by the mesenchymal-derived
BM stromal cells MBA-15 and 14F1.1, but was expressed by murine MBA-2.1 endothelial cells (Figure $2 a)$. We further found that in primary human stroma cultures, both SDF-1 and CXCR4 are expressed (Figure $2 a)$. We then tested the ability of human $\mathrm{CD} 4^{+}$cells to migrate, in a Transwell assay, in response to CM collected from different BM stromal cell lines. In correlation with the expression data, we found that CM collected from osteoblasts, expressing high levels of SDF-1 (murine MBA-15, human MG-63, and HOB3-4), induced the migration of $\mathrm{CD} 34^{+}$cells to percentages comparable to purified recombinant SDF-1 protein at $125 \mathrm{ng} / \mathrm{ml}$ (Figure 2b). The chemoattractant activity produced by the stromal cells was significantly inhibited by pretreatment of CD $34^{+}$cells with neutralizing anti-CXCR4 Ab's ( $\alpha$-CXCR4, Figure $2 b$ ) or by neutralizing CXCR4 with the T22 peptide (39) (Figure 2b), indicating that SDF-1 is responsible for the activity found in the CM. Moreover, CM from endothelial cells (MBA-2.1, HUVECs) that do not express SDF-1 mRNA, poorly attract human $\mathrm{CD}^{2} 4^{+}$cells (Figure $2 \mathrm{~b}$ ). We have shown previously that SDF-1 preferentially induces the migration of SCID repopulating cells (SRC) (14). Similarly, human $\mathrm{CD}^{2} 4^{+}$cells, which migrated in response to SDF-1 present in the CM of osteoblasts (MBA-15), were capable of repopulating the BM of NOD/SCID mice with multilineage hematopoiesis, while nonmigrating cells gave rise to only low levels of engraftment (Figure 2c). Similar results were obtained with CM from human osteoblasts (data not shown).

DNA-damaging agents increase the production of SDF-1 and the function of human SRC/stem cells. Clinical BM transplantation requires conditioning of the recipient with radiation or chemotherapy before stem cell transplantation. Similarly, most experimental transplantation protocols also involve total body irradiation (TBI) of the
Figure 3

Increased levels of SDF-1 after TBI. (a) SDF-1 mRNA expression in the BM of two NOD/SCID mice per time point. (b) Migration of human $\mathrm{CD} 34^{+}$cells to the NOD/SCID BM CM 4, 24, and 48 hours after TBI. Spontaneous migration (CTRL) and migration to SDF-1 (SDF-1). Results are mean \pm SE from seven experiments. (c) Semisolid colony assay for progenitors migrating to NOD/SCID BM CM collected before or 48 hours after TBI, without or with anti-CXCR4 Ab pretreatment. (d) Engraftment by mononuclear cells (MNC), CD $34^{+}$cells or sorted $\mathrm{CD} 34^{+} / \mathrm{CD} 38^{-/ \text {low }}$ cells transplanted immediately or 48 hours after $\mathrm{TBI}$. Mean $\pm \mathrm{SE}$ from nine experiments. $P$ values determined using student's $t$ test. GEMM, granulocyte erythrocyte macrophage megakeryocyte; GM, granulocytes-macrophages; $B F U-E$, burst forming unit erythroid. a

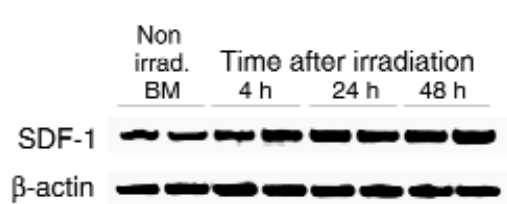

C

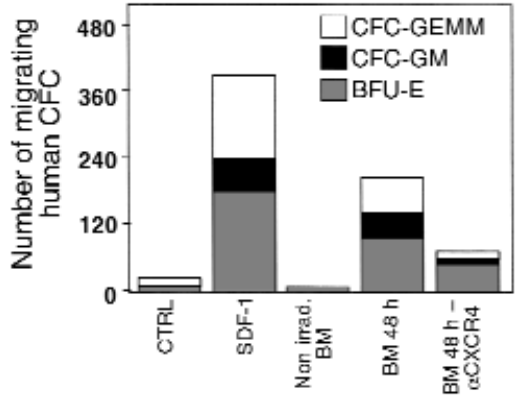

b

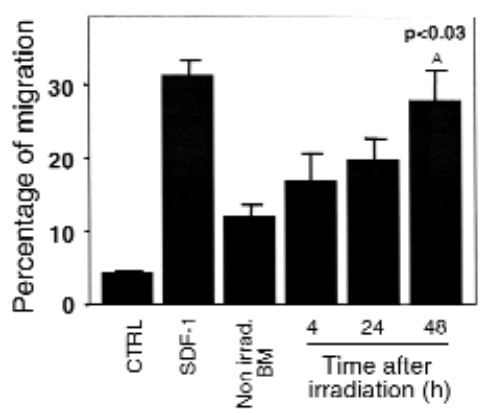

d

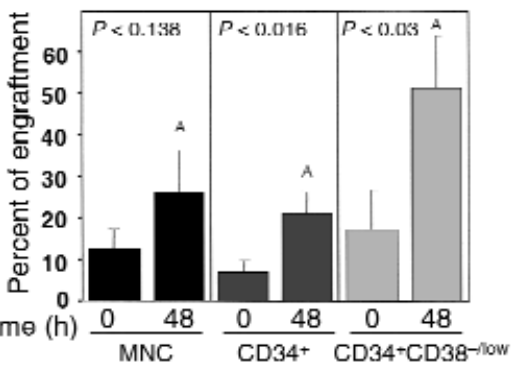




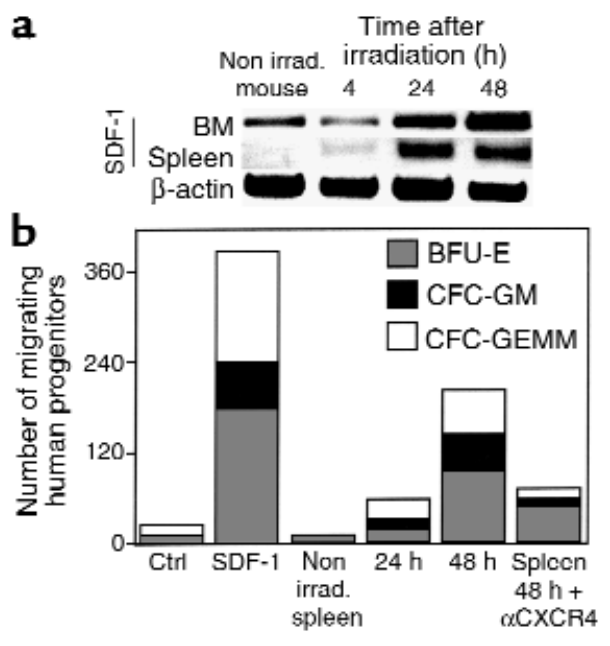

Figure 4

Increased levels of SDF-1 in the spleen of NOD/SCID after TBI. (a) SDF-1 mRNA in the BM and spleen from the same mouse per time point. (b) Semisolid colony assay for migrating progenitors to spleen CM collected before, 24 hours, or 48 hours after TBI with and without anti-CXCR4 Ab pretreatment.

recipient mice to achieve engraftment. We therefore tested the effects of irradiation on the expression of SDF-1 mRNA, using RT-PCR, and on the secretion of the chemokine, by analyzing its activity in $\mathrm{CM}$ prepared from the $\mathrm{BM}$ of $\mathrm{BALB} / \mathrm{c}, \mathrm{C} 57 \mathrm{~b} / 6$, and NOD/SCID mice before or 4, 24, and 48 hours after sublethal TBI. We found that the expression levels of SDF-1 mRNA increased significantly 24 and 48 hours after irradiation (Figure 3a). In parallel, CM showed a time-dependent increase in the potential to attract human $\mathrm{CD} 34^{+}$cells (Figure 3b). SDF-1 derived from the BM of irradiated mice induced the migration of primitive multilineage human progenitor cells in a CXCR4-dependent manner (Figure 3c). We also tested the engraftment potential of human cord blood cells immediately or 48 hours after TBI of NOD/SCID mice. One month after transplantation, mice were sacrificed, and the percentage of human cells in the mouse $\mathrm{BM}$ was assayed. When $\mathrm{CD} 34^{+}$cells were transplanted 48 hours after TBI, we found a significant $(P<0.016)$ threefold increase in the levels of engrafted cells compared with those mice transplanted immediately after irradiation (Figure 3d). Moreover, when a population enriched with more primitive human $\mathrm{CD} 34^{+} \mathrm{CD} 38^{-/ \text {low }}$ was used as donor cells, we found the highest levels of engraftment in mice transplanted 48 hours after irradiation (Figure 3d). Human engraftment in the murine $\mathrm{BM}$ included both lymphoid and myeloid cells (data not shown).

Increase in the expression of SDF-1 was also observed in the spleen of irradiated mice (Figure 4a). The increase in the expression of SDF-1 correlated well with an increase in the chemoattractant activity of the $\mathrm{CM}$ collected from spleen cells. Spleen cell-derived SDF-1 induced the migration of multilineage progenitor cells, and this migratory effect was blocked by Ab's to CXCR4 (Figure 4b).
Our data suggest that within the BM, stromal cells respond directly to irradiation with increased production of SDF-1. To test this possibility, RNA was prepared from BM stromal cell lines collected before, or 48 hours after, in vitro irradiation. By using RT-PCR analysis, we found that both mesenchymal cell lines, i.e., osteoblast (MBA-15) and in the adipocyte (14F1.1) cells, the expression levels of SDF- 1 increased 48 hours after irradiation (Figure 5a). In contrast, no increase in SDF-1 transcription after irradiation was observed when endothelial (MBA-2.1) cells that do not produce SDF-1 were examined (Figure 5a). Moreover, while the levels of SDF-1 in the CM from the stromal mesenchymal cell line MS5 increased in a dose-dependent manner in response to irradiation, irradiation had no effect on SDF-1 production by the astrocyte cell line U373, which is not from the BM stroma (Figure $5 \mathrm{~b}$ ). Interestingly, stimulation with IL- $1 \beta$ increased SDF-1 production by this cell line (Figure $5 \mathrm{~b}$ ).

DNA-damaging agents, such as Cy and 5-FU are used in a broad spectrum of clinical applications, including chemotherapy and mobilization of stem and progenitor cells from the BM into the circulation $(40,41) .5-\mathrm{FU}$ is a DNA-damaging agent that is also used for enrichment of quiescent stem cell populations in experimental transplantation protocols (42). We tested the effect of Cy and 5 -FU on the expression of SDF-1 by the BM of treated $\mathrm{BALB} / \mathrm{c}$ mice and by the murine osteoblast cell line
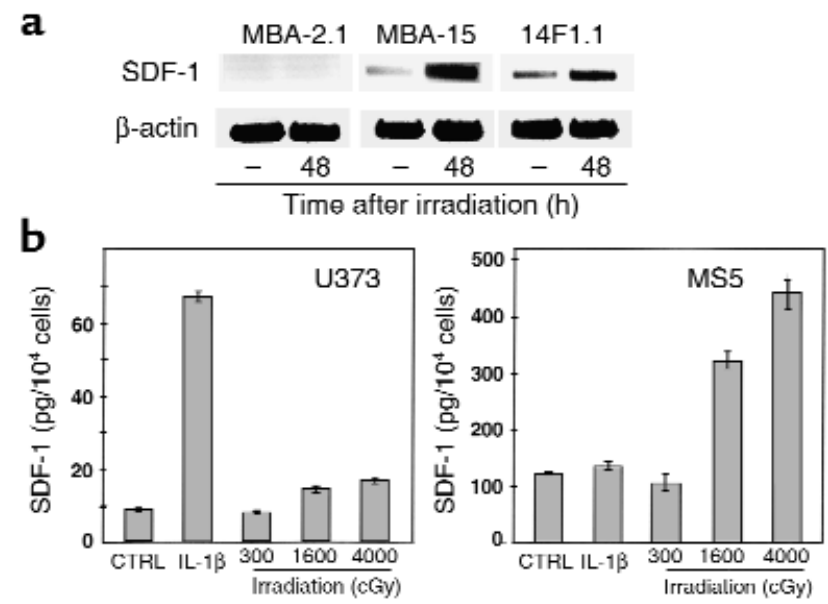

\section{C}

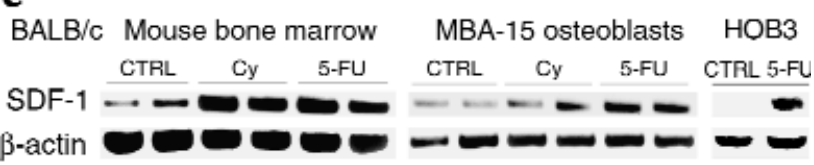

Figure 5

Influence of DNA-damaging treatment on SDF-1 expression. (a) Semiquantitative RT-PCR for murine cell lines MBA-2.1, 14F1.1, and MBA-15, before and 48 hours after 300-cGy radiation. (b) ELISA for CM of astrocytes U373 and BM mesenchymal stromal cells MS5 48 hours after stimulation with IL-1 $\beta$ (Promega Corp.) or irradiation. (c) Semiquantitative RT-PCR for SDF-1 mRNA from BM of Cyor 5-FU-treated BALB/c mice, murine MBA-15 osteoblasts (two mice or two tissue-culture plates per time point), human primary HOB3, and control levels of $\beta$-actin expression. 
MBA-15, or by human osteoblasts (HOB3). Mice treated with either Cy or 5-FU had increased levels of SDF-1 expression in the BM (Figure 5c). Similarly, murine and human osteoblasts also produced higher levels of SDF-1 in response to treatment with DNA-damaging agents (Figure 5c). Preconditioning of NOD/SCID mice 48 hours before transplantation, with 5-FU, Cy, or irradiation, increased the homing to the BM and spleen by human mononuclear cells (Figure 6, a and b). Total numbers of human $\mathrm{CD}_{4} 5^{+}$cells homing to the murine BM and spleen, were also dependent on the transplantation time after irradiation (immediately or 48 hours after TBI) (Figure 6, a and b). This effect was dependent on SDF-1, since Ab's to CXCR4 inhibited both homing and engraftment by human stem cells (ref. 14, and data not shown).

\section{Discussion}

Homing of human hematopoietic stem cells to the BM, their retention and repopulation, is essential for the establishment of intact hematopoiesis $(43,44)$. Recent reports demonstrated that the chemokine SDF-1 is essential for the repopulation of the BM by hematopoietic stem cells during murine embryonic development and in transplantation of human $\operatorname{SRC}(9,13,14,44,45)$.

In the present study we demonstrate that immature human osteoblasts, as well as stromal and endothelial cells within the human BM, express the chemokine SDF1. We found that osteoblast cell lines and mesenchymal stromal cells that are capable of supporting hematopoiesis in vitro express high levels of SDF-1. This pattern of SDF-1 expression by bone-forming cells is of great interest, since the most primitive, undifferentiated hematopoietic progenitors have been shown to be localized mostly in areas along the bone endosteum and also in periarterial regions $(46,47)$. Interestingly, we found increased expression of SDF-1 in these areas by immature osteoblasts and BM endothelial cells. It is therefore possible that early stem cells, which express CXCR4 and are known to have increased migration potential toward SDF-1, home to areas near the bone endosteum where SDF-1 is highly produced by immature osteoblasts and also to periarterial regions in which SDF-1 is produced by endothelial cells. During development, SDF-1 and its receptor CXCR4 are expressed by a wide variety of adjacent tissue pairs, including ectoderm/mesoderm and mesoderm/endothelium (2). Although CXCR4-dependent migration toward SDF-1 is increased in undifferentiated cell types, the expression and function of CXCR4/SDF-1 is also associated with differentiation of both pre-B cells and the megakaryocytic progenitors (21, 48). In addition, SDF-1 is widely needed when there is requirement for cell movement (2). Mice lacking CXCR4 have defective formation of the large vessels supplying the gastrointestinal tract and also defects in the development of the neuronal system $(13,45)$. Moreover, some endothelial and neuronal cells were shown to express CXCR4 and to migrate to SDF-1 $(13,38,49,50)$. SDF- 1 was found to synergize with VEGF in the formation of blood vessels (49). We have shown that SDF-1 can induce the arrest of rolling $\mathrm{CD}_{34}{ }^{+}$cells on human endothelium under shear flow in vitro, and that in vivo, human BM endothelial cells express SDF-1 (Figure 1; ref. 24). In agreement with our results, murine BM, but not lung endothelium, also express SDF-1 $(25,51)$. In addition, primitive progenitors also localize in periarterial regions of the $\operatorname{BM}(46,47)$.

SDF-1 is constitutively expressed by a variety of tissues, and its expression does not require proinflammatory stimulants $(2,52)$. The factors that regulate SDF-1 expression during development and in steady-state adult homeostasis are not known. Conditioning by radiation or other DNA-damaging agents is commonly used to ensure successful stem cell transplantation in the clinic. This pretreatment creates space within the BM microenvironment by eliminating host hemopoietic cells and also reduces the load of malignant cells in disease states in which the BM is afflicted. In allogeneic transplantation, irradiation also prevents the host immune response toward the graft $(1,3)$. A significant increase in mRNA levels of IL- $1 \alpha$, IL- 6 , and TNF- $\alpha$ in murine hematopoietic tissues such as the BM and spleen have been reported
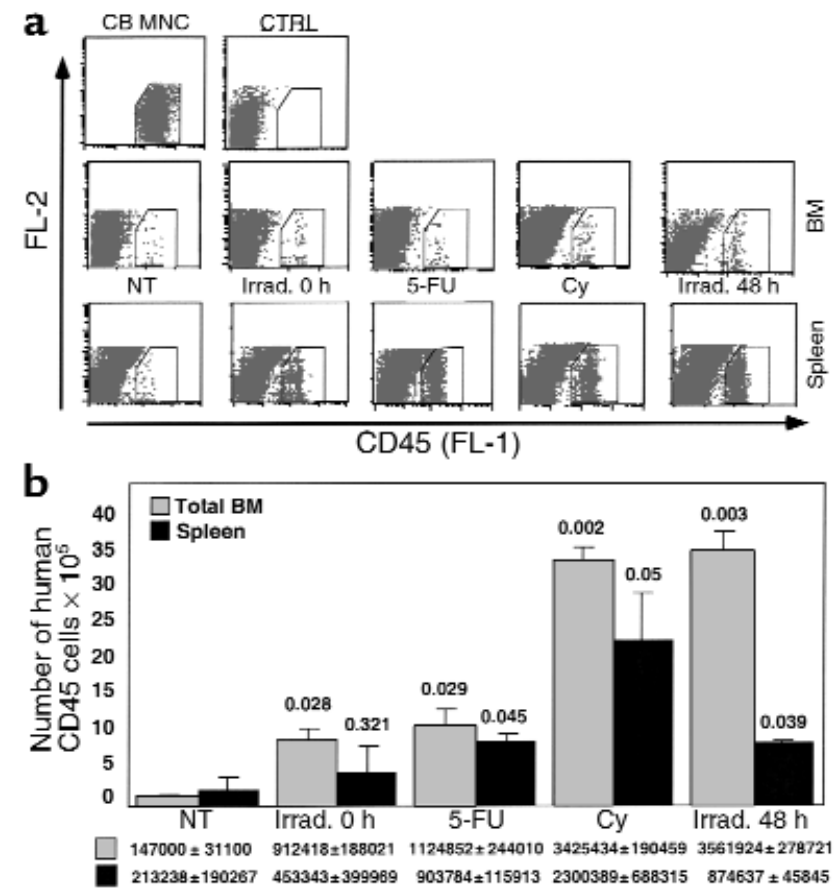

Figure 6

Increased homing of human $\mathrm{CD} 45^{+}$cells transplanted 48 hours after treatment with TBI, Cy, or 5-FU. (a) FACS plots, CD45+: CB MNC, human $C B$ MNC before transplantation; $C T R L$, nontransplanted murine BM; NT, transplantation of human CB MNC into nontreated mice; Irrad. 0 h, transplanted immediately after TBI; Irrad. 48 h, transplanted 48 hours after TBI; 5-FU or Cy, transplanted 48 hours after treatment. (b) Total numbers of human $\mathrm{CD} 45^{+}$cells in the murine BM and spleen, 24 hours after transplantation of human CB MNC $(2 \times$ $10^{7} /$ mouse) into nontreated (NT) or treated NOD/SCID mice. Results from two experiments, mean $\pm \mathrm{SE}$, are shown. Amounts of human cells were calculated for the total organ based on acquisition number of cells and cell numbers in the total organ. Numbers inside the chart represent $P$ values calculated with Student's $t$ test. FL-1, anti-CD45 FITC; FL-2, internal fluorescence. 
to occur following sublethal irradiation (53). We found that sublethal irradiation or treatment with 5-FU increases both the mRNA as well as the protein levels of SDF-1 in the BM and spleen of BALB/c, C57BL, or NOD/SCID mice. Irradiation or cytotoxic drugs also promote SDF-1 expression in human and murine bone forming osteoblasts. In parallel, the potential of human stem and progenitor cells to engraft the BM of transplanted NOD/SCID mice increases dramatically when the cells are transplanted $24-48$ hours after treatment, when the expression of SDF-1 peaks. Since SDF-1 is also a survival factor for stem cells and is involved in the development of different hematopoietic lineages, SDF-1 can also increase repopulation by promoting the survival and proliferation of transplanted stem cells. In addition, SDF-1 can also promote homing of macrophages that participate in clearing apoptotic cells and/or induce apoptosis of damaged host hematopoietic cells in the irradiated BM or spleen. Indeed, Visser et al. reported increased levels of transplanted stem cells in the liver which also produces SDF-1 and reduced levels of stem cell homing to the BM but not to the spleen in mice transplanted 4 hours after TBI in a murine stem cell-homing model compared with untreated recipients (54). In support of our work, Weiss et al. have demonstrated in a syngeneic mouse model that whole BM transplantation at limiting dilution cell doses immediately after TBI or 24 and 48 hours after TBI clearly demonstrated significantly increased levels of survival in mice transplanted 24 or 48 hours after TBI compared with mice transplanted immediately after TBI (55). Our data suggest that the increased levels of SDF-1 secreted 24-48 hours after TBI increased donor stem cell function, i.e., homing and repopulation that in turn increased the survival of the recipient mice.

Overexpression of human CXCR4 and CD4 receptors on murine T cells led to enhanced levels of these cells in the murine BM and to a dramatic decrease in their levels in the circulation (56). Furthermore, injection of SDF-1 into the murine spleen was shown to increase the homing of the murine progenitor cell line FDCP-mix cells (10). Human SDF-1 injected into the BM or spleen of nonirradiated NOD/SCID mice also attracted human SRC/stem cells in a CXCR4-dependent manner (57). Considered together with our findings, these results suggest that increase in the concentration of SDF-1 within the BM stromal microenvironment may increase the homing, retention, and repopulation of hematopoietic stem and progenitor cells. The DNAdamaging agent, 5-FU is widely used to enrich for quiescent hematopoietic stem cells (42). We found that 5 $\mathrm{FU}$, like ionizing irradiation, induces the expression of SDF-1 by murine and human BM-derived osteoblasts and significantly increases homing and engraftment of SRC. It is suggested that increase in production of SDF1 during alarm situations is part of host defense mechanism that counteracts the effects of DNA-damaging agents leading to cell death and anemia $(17,18)$. The expression of SDF-1 demonstrated here, in conjunction with irradiation and chemotherapy treatment that are commonly used in clinical protocols, may also increase repopulation by malignant cells, since many cancer cells express CXCR4 and respond to stimulation with SDF1. These include various types of leukemia cells, breast cancer cells, and prostate cancer cells (58-62). Interestingly, many malignant cells, such as multiple myeloma, and some breast cancer and prostate cancer cells, can infiltrate into the BM and in some cases also create bone lesions that could be in response to stimulation with SDF-1 secreted by the patient's BM stromal cells in response to chemotherapy $(63,64)$.

In summary, we found that immature bone-forming cells produce high levels of SDF-1, which may direct the migration and repopulation of stem cells to specific "stem cell niches" localized mostly near the endosteum and also in periarterial sites. Furthermore, we show that the expression levels of SDF-1 increase after treatment with irradiation or other DNA-damaging agents such as Cy or 5-FU and that the increase in SDF-1 production correlates well with an increase in the levels of BM homing and/or repopulation by primitive human SRC/stem cells. We therefore suggest that molecules found to increase SDF-1 production may be considered for clinical protocols with the aim of improving the outcome of stem cell transplantation provided that there are no CXCR4 ${ }^{+}$malignant cells that can also respond to stimulation with SDF-1. Our findings delineate key steps in the process of SDF-1-dependent stem cell homing/repopulation and suggest a major role for SDF-1 in alarm situations, as part of host defense processes that protect stem cells from DNA-damaging agents.

\section{Acknowledgments}

This work was supported in part by grants from Pasteur/Weizmann (to F. Arenzana-Seisdedos and T. Lapidot), The Israel Science Foundation, Concern Foundation, Israel Cancer Research Foundation (to $\mathrm{T}$. Lapidot), and NIH DE-11283 (to R. Taichman). T. Lapidot is incumbent of the Pauline Recanati Career Development Chair of Immunology.

\footnotetext{
1. Weissman, I.L. 2000. Stem cells: units of development, units of regeneration, and units in evolution. Cell. 100:157-168.

2. McGrath, K.E., Koniski, A.D., Maltby, K.M., McGann, J.K., and Palis, J. 1999. Embryonic expression and function of the chemokine SDF-1 and its receptor, CXCR4. Dev. Biol. 213:442-456.

3. Moore, M.A. 1999. "Turning brain into blood": clinical applications of stemcell research in neurobiology and hematology. N. Engl. J. Med. 341:605-607.

4. McCune, J.M., et al. 1988. The SCID-hu mouse: murine model for the analysis of human hematolymphoid differentiation and function. Science. 241:1632-1639.

5. Lapidot, T., et al. 1992. Cytokine stimulation of multilineage hematopoiesis from immature human cells engrafted in SCID mice. Science. 255:1137-1141.

6. Cashman, J.D., et al. 1997. Kinetic evidence of the regeneration of multilineage hematopoiesis from primitive cells in normal human bone marrow transplanted into immunodeficient mice. Blood. 89:4307-4316.

7. Kollet, O., et al. 2000. $\beta 2$ microglobulin-deficient ( $\beta 2 \mathrm{~m}^{\text {null }}$ ) NOD/SCID mice are excellent recipients for studying human stem cell function. Blood. 95:3102-3105

8. Larochelle, A., et al. 1996. Identification of primitive human hematopoietic cells capable of repopulating NOD/SCID mice using retroviral gene marking and cell purification: implications for gene therapy. Nat. Med. 2:1329-1337.

9. Nagasawa, T., et al. 1996. Defects of B-cell lymphopoiesis and bone-marrow myelopoiesis in mice lacking the CXC chemokine PBSF/SDF-1. Nature. 382:635-638.
} 
10. Aiuti, A., Webb, I.J., Bleul, C., Springer, T., and Gutierrez-Ramos, J.C. 1997. The chemokine SDF-1 is a chemoattractant for human CD34+ hematopoietic progenitor cells and provides a new mechanism to explain the mobilization of CD34+ progenitors to peripheral blood. J. Exp. Med. 185:111-120.

11. Bleul, C.C., Fuhlbrigge, R.C., Casasnovas, J.M., Aiuti, A., and Springer, T.A. 1996. A highly efficacious lymphocyte chemoattractant, stromal cell-derived factor 1 (SDF-1). J. Exp. Med. 184:1101-1109.

12. Jo, D.Y., Rafii, S., Hamada, T., and Moore, M.A. 2000. Chemotaxis of primitive hematopoietic cells in response to stromal cell-derived factor-1. J. Clin. Invest. 105:101-111.

13. Zou, Y.R., Kottmann, A.H., Kuroda, M., Taniuchi, I., and Littman, D.R. 1998. Function of the chemokine receptor CXCR4 in haematopoiesis and in cerebellar development. Nature. 393:595-599.

14. Peled, A., et al. 1999. Dependence of human stem cell engraftment and repopulation of NOD/SCID mice on CXCR4. Science. 283:845-848.

15. Ma, Q., Jones, D., and Springer, T.A. 1999. The chemokine receptor CXCR4 is required for the retention of $\mathrm{B}$ lineage and granulocytic precursors within the bone marrow microenvironment. Immunity. 10:463-471.

16. Kawabata, K., et al. 1999. A cell-autonomous requirement for CXCR4 in long-term lymphoid and myeloid reconstitution. Proc. Natl. Acad. Sci. USA. 96:5663-5667.

17. Lataillade, J.J., et al. 2000. Chemokine SDF-1 enhances circulating CD34(+) cell proliferation in synergy with cytokines: possible role in progenitor survival. Blood. 95:756-768.

18. Grafte-Faure, S., et al. 2000. Recruitment of primitive peripheral blood cells: synergism of interleukin 12 with interleukin 6 and stromal cell-derived factor-1. Cytokine. 12:1-7.

19. Broxmeyer, H.E., Hangoc, G., Cooper, S., and Kim, C.H. 1999. Enhanced myelopoiesis in SDF-1 transgenic mice: SDF-1 modulates myelopoiesis by regulating progenitor cell survival and inhibitory effects of myelosuppresive chemokines. Blood. 94:2886. (Abstr.)

20. Nagasawa, T., Kikutani, H., and Kishimoto, T. 1994. Molecular cloning and structure of a pre-B-cell growth-stimulating factor. Proc. Natl. Acad. Sci.USA. 91:2305-2309.

21. Hodohara, K., et al. 2000. Stromal cell-derived factor-1 (SDF-1) acts together with thrombopoietin to enhance the development of megakaryocytic progenitor cells (CFU-MK). Blood. 95:769-775.

22. Sanchez, X., et al. 1997. Activation of HIV-1 coreceptor (CXCR4) mediates myelosuppression. J. Biol. Chem. 272:27529-27531.

23. Cashman, J., Clark-Lewis, I., and Eaves, C. 2000. SDF-1 and TGF- $\beta$ enhance the detection of transplantable human stem cells regenerating in NOD/SCID mice. Exp. Hematol. 28:85. (Abstr.)

24. Peled, A., et al. 1999. The chemokine SDF-1 stimulates integrin-mediated arrest of CD34(+) cells on vascular endothelium under shear flow. J. Clin. Invest. 104:1199-1211.

25. Imai, K., et al. 1999. Selective secretion of chemoattractants for haemopoietic progenitor cells by bone marrow endothelial cells: a possible role in homing of haemopoietic progenitor cells to bone marrow. Br. J. Haematol. 106:905-911.

26. Peled, A., et al. 2000. The chemokine SDF-1 activates the integrins LFA-1, VLA- 4 and VLA- 5 on immature human CD34+ cells: role in transendothelial/stromal migration and engraftment of NOD/SCID mice. Blood. 95:3289-3296.

27. Kollet, O., et al. 2000. SDF-1 induces survival, adhesion and migration in 3D ECM like gels of murine CXCR4 null fetal liver cells via another GPCR. The American Society of Hematology. In press. (Abstr.)

28. Onai, N., et al. 2000. Impairment of lymphopoiesis and myelopoiesis in mice reconstituted with bone marrow-hematopoietic progenitor cells expressing SDF-1-intrakine. Blood. 96:2074-2080.

29. Domen, J., and Weissman, I.L. 1999. Self-renewal, differentiation or death: regulation and manipulation of hematopoietic stem cell fate. Mol. Med. Today. 5:201-208.

30. Jaffe, E.A., Nachman, R.L., Becker, C.G., and Minick, C.R. 1973. Culture of human endothelial cells derived from umbilical veins. Identification by morphologic and immunologic criteria. J. Clin. Invest. 52:2745-2756.

31. Teixido, J., Hemler, M.E., Greenberger, J.S., and Anklesaria, P. 1992. Role of beta 1 and beta 2 integrins in the adhesion of human CD34hi stem cells to bone marrow stroma. J. Clin. Invest. 90:358-367.

32. Zipori, D., Toledo, J., and von der Mark, K. 1985. Phenotypic heterogeneity among stromal cell lines from mouse bone marrow disclosed in their extracellular matrix composition and interactions with normal and leukemic cells. Blood. 66:447-455

33. Zipori, D., et al. 1985. Cultured mouse marrow stromal cell lines. II. Distinct subtypes differing in morphology, collagen types, myelopoietic factors, and leukemic cell growth modulating activities. J. Cell. Physiol. 122:81-90.

34. Robey, P.G., and Termine,J.D. 1985. Human bone cells in vitro. Calcif. Tissue Int. 37:453-460.

35. Taichman, R.S., and Emerson, S.G. 1994. Human osteoblasts support hematopoiesis through the production of granulocyte colony-stimulating factor. J. Exp. Med. 179:1677-1682.

36. Taichman, R.S., Reilly, M.J., and Matthews, L.S. 2000. Human osteoblast- like cells and osteosarcoma cell lines synthesize macrophage inhibitory protein 1alpha in response to interleukin 1beta and tumour necrosis factor alpha stimulation in vitro. Br. J. Haematol. 108:275-283.

37. Coulomb-L'Hermin, A., et al. 1999. Stromal cell-derived factor 1 (SDF-1) and antenatal human B cell lymphopoiesis: expression of SDF-1 by mesothelial cells and biliary ductal plate epithelial cells. Proc. Natl. Acad. Sci. USA. 96:8585-8590.

38. Murdoch, C., Monk, P.N., and Finn, A. 1999. CXC chemokine receptor expression on human endothelial cells. Cytokine. 11:704-712.

39. Murakami, T., et al. 1997. A small molecule CXCR4 inhibitor that blocks T cell line-tropic HIV-1 infection. J. Exp. Med. 186:1389-1393.

40. Passos-Coelho, J.L., et al. 1995. Predictive factors for peripheral-blood progenitor-cell collections using a single large-volume leukapheresis after cyclophosphamide and granulocyte-macrophage colony-stimulating factor mobilization. J. Clin. Oncol. 13:705-714.

41. Craddock, C.F., et al. 1992. Circulating stem cells in mice treated with cyclophosphamide. Blood. 80:264-269.

42. Berardi, A.C., Wang, A., Levine, J.D., Lopez, P., and Scadden, D.T. 1995. Functional isolation and characterization of human hematopoietic stem cells. Science. 267:104-108.

43. Quesenberry, P.J., and Becker, P.S. 1998. Stem cell homing: rolling, crawling, and nesting. Proc. Natl. Acad. Sci. USA. 95:15155-15157.

44. Ma, Q., et al. 1998. Impaired B-lymphopoiesis, myelopoiesis, and derailed cerebellar neuron migration in CXCR4- and SDF-1-deficient mice. Proc. Natl. Acad. Sci. USA. 95:9448-9453.

45. Tachibana, K., et al. 1998. The chemokine receptor CXCR4 is essential for vascularization of the gastrointestinal tract. Nature. 393:591-594.

46. Lambertsen, R.H., and Weiss, L. 1984. A model of intramedullary hematopoietic microenvironments based on stereologic study of the distribution of endocloned marrow colonies. Blood. 63:287-297.

47. Lord, B.I. 1990. The architecture of bone marrow cell populations. Int.J. Cell Cloning. 8:317-331.

48. Honczarenko, M., et al. 1999. SDF-1 responsiveness does not correlate with CXCR4 expression levels of developing human bone marrow B cells. Blood. 94:2990-2998.

49. Salcedo, R, et al. 1999. Vascular endothelial growth factor and basic fibroblast growth factor induce expression of CXCR4 on human endothelial cells: in vivo neovascularization induced by stromal-derived factor-1alpha. Am. J. Pathol. 154:1125-1135.

50. Bajetto, A., et al. 1999. Glial and neuronal cells express functional chemokine receptor CXCR4 and its natural ligand stromal cell-derived factor 1. J. Neurochem. 73:2348-2357.

51. Imai, K., et al. 1999. Selective transendothelial migration of hematopoietic progenitor cells: a role in homing of progenitor cells. Blood. 93:149-156.

52. Pablos, J.L., et al. 1999. Stromal-cell derived factor is expressed by dendritic cells and endothelium in human skin. Am. J. Pathol. 155:1577-1586.

53. Chang, C.M., et al. 1997. Sublethal gamma irradiation increases IL-1alpha, IL-6, and TNF-alpha mRNA levels in murine hematopoietic tissues. J. Interferon Cytokine Res. 17:567-572.

54. Hendrikx, P.J., Martens, C.M., Hagenbeek, A., Keij,J.F., and Visser,J.W. 1996. Homing of fluorescently labeled murine hematopoietic stem cells. Exp. Hematol. 24:129-140.

55. Weiss, L., Bullorsky, E., Ashkenazi, Y.J., and Slavin, S. 1988. Optimal time interval between myeloablative whole body irradiation and reconstitution with syngeneic bone marrow graft. Bone Marrow Transplant. 3:207-210.

56. Sawada, S., et al. 1998. Disturbed CD4+ T cell homeostasis and in vitro HIV1 susceptibility in transgenic mice expressing T cell line-tropic HIV-1 receptors. J. Exp. Med. 187:1439-1449.

57. Kollet, O., Peled, A., and Lapidot, T. 1999. Exclusive homing of human CD $38^{-/ \text {low }} \mathrm{CXCR}^{+}$stem cells to the spleen and bone marrow of immune deficient mice within 1-16 hours. Blood. 94:1731. (Abstr.)

58. Sehgal, A., Keener, C., Boynton, A.L., Warrick, J., and Murphy, G.P. 1998. CXCR-4, a chemokine receptor, is overexpressed in and required for proliferation of glioblastoma tumor cells. J. Surg. Oncol. 69:99-104.

59. Bradstock, K.F., et al. 2000. Effects of the chemokine stromal cell-derived factor- 1 on the migration and localization of precursor-B acute lymphoblastic leukemia cells within bone marrow stromal layers. Leukemia. 14:882-888.

60. Mohle, R., et al. 1998. The chemokine receptor CXCR-4 is expressed on CD34+ hematopoietic progenitors and leukemic cells and mediates transendothelial migration induced by stromal cell-derived factor-1. Blood. 91:4523-4530.

61. Koshiba, T., et al. 2000. Expression of stromal cell-derived factor 1 and CXCR4 ligand receptor system in pancreatic cancer: a possible role for tumor progression. Clin. Cancer Res. 6:3530-3535.

62. Mitra, P., et al. 1999. CXCR4 mRNA expression in colon, esophageal and gastric cancers and hepatitis C infected liver. Int. J. Oncol. 14:917-925.

63. Lalle, M., De Rosa, L., Marzetti, L., and Montuoro, A. 2000. Detection of breast cancer cells in the bone marrow or peripheral blood: methods and prognostic significance. Tumori. 86:183-190.

64. Zhau, H.E., Li, C.L., and Chung, L.W. 2000. Establishment of human prostate carcinoma skeletal metastasis models. Cancer. 88:2995-3001. 